\title{
Fortalecimento da inserção política e socio familiar do idoso: o papel dos grupos de apoio no desenvolvimento da autonomia e independência
}

\author{
Strengthening the political and socio familiary institution of the elderly: the role of support groups in \\ the development of autonomy and independence
}

Fortalecimiento de la inserción política y sociofamiliar del idoso: el papel de los grupos de apoyo en el desarrollo de la autonomía e independencia

Gabriel Felipe Lyra Silva ${ }^{1}$, Israela Cristine Pereira Marinho', Juliana Joyce Chaves de Lima ${ }^{1}$, Lorena Batista de Lemos $^{1}$, Marina Lopes de Freitas Freire ${ }^{1 *}$, Mariseth de Carvalho Andrade ${ }^{2,3,5}$, Osvaldo da Silva Peixoto ${ }^{1,2,3,4}$, Yanka Rafaela da Costa Neto Vieira ${ }^{1}$.

\section{RESUMO}

Objetivos: Integrar a comunidade de idosos da USF da Visconde por meio da participação em grupos de novas atividades multidisciplinares. $O$ estudo visa também avaliar o desempenho cognitivo, o índice de depressão e declínio funcional através da aplicação de testes específicos. Métodos: Trata-se de um estudo longitudinal, de base populacional, com intervenção em participantes, sendo o público alvo, idosos cadastrados na USF da Visconde e que possuam capacidade funcional de se deslocar até a unidade. Foram realizados 6 encontros, nos quais estava presente um profissional que realizou palestras e oficinas com os idosos. Foram aplicados três questionários: MEEM, Caracterização biossocial, familiar, econômica e de saúde e WHOQOL-OLD. A tabulação estatística foi realizada usando Microsoft Excel e Biostat 5.0 e utilizado níveis de significância de 95\%. Resultados: Foram incluídos 28 pacientes no estudo, sendo a média de idade 68.6 anos. Todos alegaram possuir uma doença crônica, sendo a hipertensão arterial sistêmica a mais frequente $(66,7 \%)$. O estudo teve impacto positivo sobre a "Autonomia, Passado, presente e futuro, Participação social e a Qualidade de vida". Antes da intervenção, indivíduos que praticavam exercício físico tiveram, maior índice de qualidade de vida avaliado pelo WHOQOL quando comparados aos indivíduos que não praticam. Conclusão: O projeto obteve resultados satisfatórios, alcançando integração comunitária entre idosos e manutenção da capacidade funcional, por meio da participação nos grupos de apoio. Portanto, essas ações de fortalecimento socio familiar e político da população idosa são fundamentais para melhorar o desempenho cognitivo, os índices de depressão e até mesmo o declínio funcional.

Palavras-chave: Idoso, Qualidade de vida, Relações interpessoais.

\begin{abstract}
Objectives: To integrate Visconde's FHU senior's community through participation in groups of new multidisciplinary activities. The study also aims to evaluate the cognitive performance, depression index, and functional decline through the application of specific tests. Methods: This is a longitudinal, population-based study with intervention in participants, being the target audience, elderly people registered in the Visconde's $\mathrm{FHU}$ and who have the functional capacity to move to the unit. Six meetings were held, in which a professional was present who gave lectures and workshops with the elderly present. Three questionnaires were applied: MMSE, biossocial characterization, family, economic and health, and WHOQOL-OLD. The statistical tabulation was performed using Microsoft Excel and Biostat 5.0 and significance levels of $95 \%$ were used.
\end{abstract}

${ }^{1}$ Faculdade Metropolitana da Amazônia, Belém-PA.

Universidade Federal do Pará, Belém-PA.

${ }^{3}$ Universidade do Estado do Pará, Belém-PA.

${ }^{4}$ Pontifícia Universidade Católica do Rio de Janeiro, Rio de Janeiro-RJ.

${ }^{5}$ Santa Casa de Misericórdia do Pará, Belém-PA. *E-mail: marinafreitasfreire@gmail.com 
Results: 28 patients were included in the study and the mean age was 68.6 years. All of them claimed to have a chronic disease, with systemic arterial hypertension being the most frequent (66.7\%). The study had a positive impact on "Autonomy, Past, Present and Future, Social Participation and Quality of Life". Before the intervention, individuals who practiced physical exercise had a higher quality of life index assessed by the WHOQOL when compared to individuals who did not practice. Conclusion: The project achieved satisfactory results, achieving community integration among the elderly and maintaining functional capacity, through participation in support groups. Therefore, these actions of socio-family and political strengthening of the elderly population are fundamental to improve cognitive performance, indices of depression and even functional decline.

Key words: Elderly, Quality of life, Interpersonal relations.

\section{RESUMEN}

Objetivos: Integrar la comunidad de ancianos de la USF de la Visconde a través de la participación en grupos de nuevas actividades multidisciplinares. El estudio pretende también evaluar el desempeño cognitivo, el índice de depresión y declinación funcional a través de la aplicación de pruebas específicas. Métodos: Se trata de un estudio longitudinal, de base poblacional, con intervención en participantes, siendo el público objetivo, ancianos registrados en la USF de la Visconde y que poseen capacidad funcional de desplazarse hasta la unidad. Se realizaron 6 encuentros, en los que estaba presente un profesional que realizó conferencias y talleres con los ancianos presentes. Se aplicaron tres cuestionarios: MEEM, Caracterización biossocial, familiar, económica y de salud y WHOQOL-OLD. La tabulación estadística se realizó utilizando Microsoft Excel y Biostat 5.0 y se utilizaron niveles de significancia del 95\%. Resultados: Se incluyeron 28 pacientes en el estudio, siendo el promedio de edad 68.6 años. Todos alegaron poseer una enfermedad crónica, siendo la hipertensión arterial sistémica la más frecuente $(66,7 \%)$. El estudio tuvo un impacto positivo sobre la "Autonomía, Pasado, presente y futuro, Participación social y la Calidad de vida". Antes de la intervención, individuos que practicaban ejercicio físico tuvieron, mayor índice de calidad de vida evaluado por el WHOQOL cuando comparados a los individuos que no practican. Conclusión: El proyecto obtuvo resultados satisfactorios, alcanzando integración comunitaria entre ancianos y mantenimiento capacidad funcional, por medio de la participación en los grupos de apoyo. Por lo tanto, estas acciones de fortalecimiento sociofamiliar y político de la población anciana son fundamentales para mejorar el desempeño cognitivo, los índices de depresión e incluso el declive funcional.

Palabras clave: Ancianos, Calidad de vida, Relaciones interpersonales.

\section{INTRODUÇÃO}

O envelhecimento populacional representa um fenômeno universal, característico tanto dos países desenvolvidos como países em desenvolvimento. Devido a esta revolução demográfica, estima-se que os idosos representarão um quarto da população mundial até o ano de 2025. (BORGES MCM, 2006).

A estruturação da pirâmide etária brasileira enquadra-se nesses padrões e demonstra significativas mudanças com o passar dos anos. No século 20 , o aumento da expectativa de vida da população em 30 anos foi o acontecimento mais significativo nesse âmbito, revolucionando o cenário demográfico e o contexto da saúde pública brasileira. A diminuição da mortalidade e da fecundidade somado ao desenvolvimento tecnológico na área da saúde tem sido apontado como fundamentais neste processo de envelhecimento mundial. A estimativa é que em 2025 o Brasil seja o sexto país mais envelhecido do mundo, com uma média de 32 milhões de pessoas com mais de 60 anos, o que representará 15\% da população nacional. (VILELA ABA, 2006).

Devido ao novo cenário demográfico brasileiro, no qual a expectativa de vida vem sendo prolongada, é necessário também proporcionar à população idosa uma melhor qualidade de vida e garantia aos serviços básicos de saúde. Nesse contexto, deve-se compreender o envelhecimento como um processo dinâmico e 
progressivo, determinado tanto por alterações morfológicas, funcionais e bioquímicas, quanto por modificações psicológicas (BORGES MCM, 2006; ALBUQUERQUE ,2011).

Diante disso, o envelhecimento natural está ligado à capacidade de adaptação do indivíduo aos rigores e às agressões do meio ambiente; por outro lado, o desenvolvimento de uma condição patológica por estresse emocional, acidente ou doenças é caracterizado como senilidade. Essas alterações podem influenciar na capacidade de adaptação ao meio, principalmente de uma forma progressiva e negativa, deixando esse idoso mais vulnerável a patologias que podem ter um desfecho letal. A perda da capacidade funcional é uma das principais adversidades de saúde relacionadas à idade, podendo vir acompanhada de dependência física, ocupacional ou social e ainda limitação de funções e atividades relativas à vida diária (VILELA ABA, 2006).

A autonomia dos idosos está intimamente relacionada à manutenção das atividades da vida diária (AVD), que são domínios básicos de autocuidado, e às atividades instrumentais da vida diária (AIVD), que se situam como competências necessárias para se viver de maneira independente. O cuidado com tais necessidades citadas preserva a capacidade funcional do indivíduo. Para a população idosa realizar as AVD é algo fundamental para o autocuidado e preservação da saúde; já as AIVD são importantes para manter o indivíduo participativo socialmente. (FERREIRA OGL et al, 2012).

A busca por uma melhor qualidade de vida é um dos principais objetivos da população idosa, visto que, viver mais não é viver melhor. A não realização das AVD e AIVD podem deixar o idoso dependente e isolado, gerando patologias como depressão e isolamento do convívio e participação social, tornando-o improdutivo. A saúde pública tem em seus preceitos básicos os direitos da população idosa; por isso estão sendo desenvolvidos programas sociais que visam a preservação da independência e da autonomia, como a Estratégia de Saúde da Família (ESF), desenvolvida pelas equipes das Unidades Básicas de Saúde. A ESF atua no cenário da Atenção Básica, aproximando os idosos das comunidades por meio de ações multidisciplinares, que levam em consideração o cenário no qual o idoso se encontra e suas peculiaridades, valorizando o idoso e seu papel social e familiar. (OLIVEIRA LPBA e MENEZES MP, 2012; WALTER MMT, 2010).

Verifica-se que as estratégias envolvendo o idoso têm o propósito de respeitá-los, assim como às suas famílias, reconhecendo-os como sujeitos se sua própria história e isso é de fundamental importância para seu acolhimento socio familiar. Sendo assim, busca-se atingir os objetivos citados trabalhando com grupos de indivíduos, na expectativa de promover o aumento da consciência crítica por intermédio da troca de ideias entre os sujeitos. A estratégia de grupos pode contribuir na promoção, proteção e controle de doenças. Constata-se que as reuniões são um espaço complementar de troca de informações e estímulo social (MOLINI-AVEJONAS DR et al, 2010).

Na saúde coletiva, o grupo é uma estratégia de trabalho e ao mesmo tempo de investigação das condições dos usuários do Sistema Único de Saúde. Por meio deles, pode-se oferecer educação em saúde, com o objetivo de criar ambientes favoráveis ao desenvolvimento humano pela adoção de hábitos e estilos de vida saudáveis que tragam mais qualidade de vida à população. As atividades de grupos facilitam o exercício da autodeterminação e da independência, pois podem funcionar como rede de apoio que mobiliza as pessoas na busca de autonomia e sentido para a vida e na melhora da autoestima. Neste contexto e diante destes benefícios, a formação de grupos de convivência de idosos vem sendo utilizada para proporcionar aos mesmos a preservação da capacidade funcional que mantém sua autonomia e independência, reintegrandoos efetivamente no contexto socio familiar (GARCIA MMA et al, 2006).

Diante de todos os fatos citados, faz-se necessário a interação entre os idosos das comunidades e seus familiares e à sociedade na qual estão inseridos, a fim de melhorar suas atividades físicas, cognitivas e mentais, garantindo autonomia, independência e completo exercício de sua cidadania. Esse trabalho objetiva integrar a comunidade de idosos da Unidade de Saúde da Família Canal da Visconde por meio da participação em grupos de novas atividades multidisciplinares, que busquem a melhoria da qualidade de vida, assim como, avaliar o perfil sociodemográfico dos idosos da comunidade, o desempenho cognitivo, o índice de depressão e declínio funcional através da aplicação de testes específicos. 


\section{METODOLOGIA}

O presente estudo é do tipo longitudinal, sendo realizado segundo os preceitos da Declaração de Helsinque e do Código de Nuremberg em uma Unidade de Saúde da Família (USF) em Belém, respeitando as normas de pesquisas envolvendo seres humanos (Res. CNS 466/12) do Conselho Nacional de Saúde e após a aprovação do projeto pelo Comitê de Ética em Pesquisa (número do parecer: 2.387.465), pela instituição responsável pelos dados e autorização dos pacientes ou responsáveis, por meio de termo de consentimento livre e esclarecido (TCLE).

Inicialmente, os acadêmicos de Medicina e médico responsável, realizaram uma palestra na Unidade de Saúde da área, na qual foi apresentado às equipes de saúde da USF o projeto de intervenção informando-os quanto a importância e objetivos que se pretendia alcançar. Neste dia, também desejou-se sensibilizar e integrar as equipes objetivando um maior comprometimento na iniciativa de consolidar o projeto. Além disso, foi promovido uma capacitação aos agentes comunitários de saúde (ACS) sobre conceitos de envelhecimento, necessidades e dificuldades inerentes à terceira idade; enfocando-se a assistência necessária no acolhimento do idoso na instituição e sua importância para o desenvolvimento do projeto.

Os sujeitos que aceitaram participar do projeto foram orientados a comparecer mensalmente a unidade de saúde junto a algum membro da família, onde receberam acompanhamento pelos participantes do projeto durante 03 meses após início dos encontros.

Os encontros ocorreram duas vezes ao mês, em dias fixos pela manhã na unidade de saúde, em salas destinadas para os acadêmicos de medicina. Nos encontros, estava presente um profissional capacitado e especializado na área de geriatria, este foi disponibilizado pela Secretaria Municipal de Saúde em parceria com o SESI (Serviço Social da Indústria).

Durante as reuniões foram desenvolvidas dinâmicas, vivências, palestras e oficinas, trabalhando temas como: estatuto do idoso, a valorização e inclusão social do idoso, mitos da velhice, relações interpessoais e familiares, maturidade com qualidade de vida e atividades de estimulação cognitiva, dentre outros temas fundamentais para conhecimento do idoso.

As atividades desenvolvidas nas dinâmicas e oficinas foram: oficinas de memória e palestra explicativa sobre Alzheimer e suas repercussões, jogos que estimularam uma maior interação entre os participantes, palestras sobre a importância do idoso na sociedade e seus direitos e deveres, discussões a respeito da valorização do idoso no seio familiar, rodas de conversa abertas e respeitosas, nas quais foi possível ouvir as queixas mais frequentes dessa população, debates sobre os mitos da velhice e a maturidade com qualidade de vida, dinâmicas ativas que estimularam a movimentação dos idosos e que valorizaram a participação e a fala e esclarecimentos sobre o Estatuto do Idoso.

Foram aplicados no primeiro e no último encontro três questionários: Mini Exame do Estado Mental; Caracterização biossocial, familiar, econômica e de saúde; Questionário Whoqol-Old.

O estudo e tabulação estatística dos dados obtidos foram realizados de acordo com a natureza das variáveis e usando como ferramentas softwares específicos (Microsoft Excel, Biostat 5.0), utilizando-se também de estatística descritiva. As variáveis contínuas foram expressas sob a forma de média com ajuste por desvio padrão e as categóricas foram expressas sob a forma de frequência absoluta e relativa. Os resultados obtidos foram expressos sob a forma de gráficos e/ou tabelas. foi utilizado níveis de significância de $95 \%$ nas avaliações estatísticas.

\section{RESULTADOS E DISCUSSÃO}

Foram identificados, durante o período do estudo, 28 pacientes que atendiam aos critérios de inclusão definidos, os quais foram convidados a participar da presente pesquisa. Destes, a maioria $(18 / 28,64,3 \% ; p=$ $0.1859)$ era do sexo feminino, casados ou em união estável $(10 / 28,35,7 \% ; p<0.00001)$, com uma média de idade de 68.6 anos (+6.9), variando entre 60 e 83 anos. A tabela 1 apresenta o perfil sociodepidemológico dos indivíduos incluídos no estudo. 
O fato da maioria dos participantes do estudo serem mulheres (Tabela 1) iguala-se a dados nacionais de predominância feminina acerca de cuidados e valores de atenção à saúde. A mulheres procuram mais os serviços de saúde em relação sexo oposto; muitos homens que situam-se como base familiar ou como provedor da família, acreditam que não devem procurar serviços de saúde para não demonstrar fraqueza, assim o processo saúde-doença para os homens é bastante vinculada ao aspecto curativista, ao passo que para mulheres é ligada à prevenção, essa informação também contribui para esclarecer a maior colaboração feminina no estudo (FERREIRA FPC, 2014).

Além disso, as condições de saúde são fortemente relacionadas à renda familiar, sendo a aposentadoria um dos marcos da velhice; com o afastamento do trabalho, pode haver uma redução da participação social, enfraquecendo relações antes fortalecidas pela rotina ativa Dados do IBGE mostram que a maior parte da renda é proveniente de aposentadorias: $65 \%$ das pessoas de 60 anos e mais e $71 \%$ das pessoas de mais de 75 anos têm essa como a única fonte de renda (PAOLINI KS, 2016; RIBEIRO GG et al, 2015).

No presente estudo, também se comprovou que $35,7 \%$ encontram-se aposentados e deixaram de trabalhar, e que $10,7 \%$ se aposentaram, mas ainda trabalham de alguma forma; além disso, a maioria (46,3\%) ainda exerce alguma atividade trabalhista, seja ela empregatícia, autônoma ou por outras formas; e que apenas $7,1 \%$ encontra-se desempregada (Tabela 1). Assim, percebe-se que a maioria dos idosos do estudo ainda possui, direta ou indiretamente, uma vida economicamente produtiva. Isso corrobora com estudos que apontam o idoso como um apoio familiar de grande importância devido à renda de suas aposentadorias ou trabalhos informais, visto que a quantidade de idosos chefes de família tem aumentado ano a ano (RIBEIRO GG et al, 2015).

Tabela 1 - Perfil socio epidemiológico dos usuários cadastrados na Unidade de Saúde da Família Canal da Visconde que participaram do projeto de intervenção entre dezembro/2017 a março/2018.

\begin{tabular}{|c|c|c|}
\hline Perfil Socioeconômico & n (\%) & $p$-valor \\
\hline Sexo & & \multirow{3}{*}{0.1859} \\
\hline Feminino & $18(64,3)$ & \\
\hline Masculino & $10(35,7)$ & \\
\hline \multicolumn{3}{|l|}{ Idade } \\
\hline 60 a 64 & $11(39,3)$ & \multirow{7}{*}{$<0.00001^{*}$} \\
\hline 65 a 69 & $4(14,3)$ & \\
\hline 70 a 74 & $6(21,4)$ & \\
\hline 75 a 79 & $5(17,9)$ & \\
\hline$>=80$ & $2(7,1)$ & \\
\hline Média \pm de idade & \multirow{3}{*}{$\begin{array}{c}68.6 \pm 6.9 \\
60.0 / 68.0 / 83.0\end{array}$} & \\
\hline Mínimo / Mediana / Máximo & & \\
\hline Estado civil & & \multirow{5}{*}{$<0.00001^{*}$} \\
\hline Solteiro & $4(14,3)$ & \\
\hline Casado/União estável ${ }^{*}$ & $11(39,3)$ & \\
\hline Divorciado & $5(17,9)$ & \\
\hline Viúvo & $8(28,6)$ & \\
\hline \multicolumn{3}{|l|}{ Religião } \\
\hline Católico & $15(53,6)$ & \multirow{3}{*}{$<0.00001$} \\
\hline Evangélico & $11(39,3)$ & \\
\hline Espírita & $2(7,1)$ & \\
\hline
\end{tabular}




\section{Escolaridade}

Nunca estudou

Fundamental incompleto

$8(27,6)$

Fundamental Completo

$4(13,8)$

$<0.00001$

Médio incompleto

$5(17,2)$

Médio Completo

$8(27,6)$

Superior incompleto

\section{Número de filhos}

Não possui

01 a 02

$8(28,6)$

03 a 04

$6(21,4)$

0.5853

05 a 06

07 ou mais

$7(25,0)$

$5(7,9)$

\section{Situação atual de trabalho}

$\begin{array}{lc}\text { Empregado } & 9(32,1) \\ \text { Autônomo } & 2(7,1) \\ \text { Desempregado } & 2(7,1) \\ \text { Aposentado, mas continua trabalhando } & 3(10,7) \\ \text { Aposentado e deixou de trabalhar } & 10(35,7) \\ \text { Outras } & 2(7,1)\end{array}$

Teste Qui-Quadrado de Aderência.

*Teste G de Aderência.

Fonte: Protocolo de Pesquisa, 2018.

Quanto a percepção de saúde, os participantes, em sua maioria $(12 / 28,42,9 \% ; p<0.00001)$, a caracterizaram como regular e se comparados com outras pessoas da mesma idade, a referiram como melhor (15/28, 53,6\%; $p<0.00001)$; não houve incidência significativa na avaliação da saúde em comparação ao ultimo ano. Todos os participantes alegaram possuir uma doença crônica, sendo a hipertensão arterial sistêmica a mais frequente $(18 / 28,66,7 ; p<0.00001)$, apenas $10,7 \%(3 / 28)$ não fazem o uso adequados dos remédios. Houve incidência significante de pacientes que referem serem tratados pelos filhos em caso de incapacidade (17/28, 60,7\%; $p<0.00001)$ (Tabela 2).

Tabela 2 - Percepção de saúde dos usuários cadastrados da Unidade de Saúde da Família Canal da Visconde que participaram do projeto de intervenção entre dezembro/2017 a março/2018.

\begin{tabular}{lcl}
\hline Em geral como o(a) Sr.(a) diria que está a sua saúde? & \\
\hline \hline Ótima & 4 & $14,3 \%$ \\
Muito boa & 1 & $3,6 \%$ \\
Boa & 9 & $32,1 \%$ \\
Regular* & 12 & $42,9 \%$ \\
Ruim & 2 & $7,1 \%$ \\
\hline Total & $\mathbf{2 8}$ & $\mathbf{1 0 0 , 0 \%}$
\end{tabular}

$\overline{\mathrm{P}<0.00001 \text { (Teste G de Aderência). }}$

Fonte: Protocolo de pesquisa, 2018. 
O envelhecimento relaciona-se a mudanças fisiológicas, sociais e psicológicas que interferem na capacidade funcional, podendo influenciar nas atividades de vida diária e na inserção familiar do idoso, contribuindo para o surgimento de Doenças Crônicas Não Transmissíveis (DCNT), principalmente doenças cardiovasculares e diabetes mellitus (DM) (SILVA JVF et al, 2015). Esse fato comprovou-se no estudo, visto que $100 \%$ dos idosos participantes possuíam alguma patologia de base, sendo a Hipertensão Arterial Sistêmica (HAS), seguida da Diabetes Mellitus (DM) e do hipotireoidismo (Tabela 3).

Devido a esses dados, foi realizada palestra sobre o tema "Repercussão da alimentação balanceada no idoso", visto que o papel do equilíbrio alimentar pode repercutir no agravamento ou no controle de tais patologias. Após a intervenção, os idosos realizaram uma roda de conversa a respeito das informações relatadas, obtendo um bom desempenho.

Tabela 3 - Perfil de doenças crônicas dos usuários cadastrados da Unidade de Saúde da Família Canal da Visconde que participaram do projeto de intervenção entre dezembro/2017 a março/2018.

\begin{tabular}{ccc}
\hline Doença Crônica & Frequência & $\%$ (N = 28) \\
\hline \hline É portador & 27 & $96,4 \%$ \\
Sim & 1 & $3,6 \%$ \\
Não & & $\mathbf{N}=\mathbf{2 7}$ \\
Qual(is) & 18 & $66,7 \%$ \\
HAS & 4 & $14,8 \%$ \\
DM & 2 & $7,4 \%$ \\
HAS + DM & 2 & $7,4 \%$ \\
HAS + DM + Hipotireoidismo & 2 & $7,4 \%$ \\
Outras & & $\mathbf{N}=\mathbf{2 7}$ \\
Tempo de doença (anos) & 11 & $40,7 \%$ \\
Menos de 10 & 6 & $22,2 \%$ \\
10 a 19 & 8 & $29,6 \%$ \\
20 ou mais & 25 & $7,4 \%$ \\
Não sabe informar & 3 & $89,3 \%$ \\
Sim & $25,7 \%$ \\
Nõo & &
\end{tabular}

$P<0.00001$ (Teste G de Aderência).

Fonte: Protocolo de pesquisa, 2018.

Foi observado que o projeto de intervenção teve relevância nos participantes após a avalição do Mini Exame do Estado Mental, visto que a porcentagem de idosos com defeitos cognitivos diminuiu de $35,7 \%$ (10/28) para 21,4\% (6/28) (Tabela 4). 
Tabela 4 - Avaliação do Mini Exame do Estado Mental dos usuários cadastrados da Unidade de Saúde da Família Canal da Visconde que participaram do projeto de intervenção entre dezembro/2017 a março/2018.

\begin{tabular}{ccccc}
\hline MMSE & & Primeira Avaliação & \multicolumn{2}{c}{ Segunda Avaliação } \\
\hline \hline Com defeito cognitivo & 10 & $35,7 \%$ & 6 & $21,4 \%$ \\
Sem defeito cognitivo & 18 & $64,3 \%$ & 22 & $78,6 \%$ \\
\hline
\end{tabular}

$p=0.0247$ (Teste Qui-Quadrado Aderência).

Fonte: Protocolo de pesquisa, 2018.

Após a avaliação de WHOQOL-OLD antes e após a intervenção, foi possível notar que a intervenção proposta pelo presente estudo teve impacto positivo sobre a Autonomia (teste t pareado, $t=2.750, \mathrm{GL}=26$, $p=0.0105$ ), Passado, presente e futuro (teste t pareado, $t=4.204, G L=26, p=0.0003$ ), Participação social (teste $t$ pareado, $\mathrm{t}=6.913, \mathrm{GL}=26, \mathrm{p}<0.0001$ ) e a Qualidade de vida (teste t pareado, teste $\mathrm{t}, \mathrm{t}=6.057, \mathrm{GL}$ $=26, p<0.0001$ ).

É importante ressaltar, que o estado de saúde está relacionado à qualidade de vida, por isso, alguns autores questionaram se a presença de comorbidades, por si só, levaria a um indivíduo considerar-se menos saudável. Ferraz e Peixoto verificaram "que o idoso pode apresentar uma percepção positiva da saúde desde que seus problemas não signifiquem limitações da sua autonomia, seja como indivíduo, seja enquanto ser social'. Outros estudos sobre o grau de satisfação com a saúde entre idosos do Brasil demonstraram que o percentual que considera sua saúde "boa" cresceu de 34\% para 41\% (ALBERTE JS et al, 2015).

Um fato que justifique essa a melhora da avaliação da própria saúde é a prática de atividade física, que combate o sedentarismo e contribui para a manutenção da capacidade funcional (GUIMARÃES RA e AMÉLIO SAA, 2018). Por esse motivo, durante a intervenção foi feita uma palestra a respeito da "Influência da atividade física na saúde do idoso", e após isso, foi realizada roda de conversa para expor dificuldades encontradas na comunidade para prática de exercício.

Diferentemente dos estudos nacionais, nesse projeto, antes da intervenção, a maioria dos idosos consideravam seu estado de saúde como "regular" (42,9\%) e apenas 31,1\% o considerava "bom", pôde-se relacionar que os idosos que consideraram "boa" qualidade de vida eram praticantes de exercício físico. Outro resultado importante do estudo, foi que, após a intervenção, a parcela que não praticava nenhuma atividade física mostrou aumento da qualidade de vida, ou seja, a intervenção feita pelo grupo teve a mesma influência que a prática de atividade física nos idosos estudados (Tabela 5).

Tabela 5 - Relação entre a prática de exercício físico e o desempenho em WHOQOL-OLD antes e após a intervenção.

\begin{tabular}{cccccc}
\hline Avaliação & $\begin{array}{c}\text { Prática } \\
\text { exercício }\end{array}$ & $\begin{array}{c}\text { Não prática } \\
\text { exercício }\end{array}$ & $\begin{array}{c}\text { Diferença entre } \\
\text { as médias }\end{array}$ & $\mathrm{t}$ & $\mathrm{p}$ \\
\hline $\begin{array}{c}\text { QV antes da } \\
\text { intervenção }\end{array}$ & $4.221 \pm 0.1262$ & $3.770 \pm 0.1703$ & 0.4512 & 2.168 & $0.039^{*}$ \\
$\begin{array}{c}\text { QV após a } \\
\text { intervenção }\end{array}$ & $4.330 \pm 0.1086$ & $3.973 \pm 0.1508$ & 0.3573 & 1.970 & $0.059^{*}$ \\
\hline
\end{tabular}

${ }^{\star}$ Teste T pareado.

Fonte: Protocolo de pesquisa, 2018. 
No Brasil, as redes de apoio social são muito escassas, sendo um dos obstáculos ao se atender às necessidades dos idosos refletindo na institucionalização de idosos Além disso, parte dessas instituições não dispõe de profissionais suficientes e qualificados para atenção ao idoso. Outro ponto negativo, é que esse fato ocasiona distanciamento dos membros familiares, diminuição da rede social dos idosos, aumento da vulnerabilidade dessa população e até mesmo maior suscetibilidade às doenças (AMARAL LF et al, 2013; LEITÃO MCM et al, 2016). Por esse motivo, o grupo atuou intervindo nesses postos-chave necessários à população geriátrica. Os dados do estudo, após a intervenção, mostraram aumento na autonomia dos idosos participantes desse grupo de apoio.

Esses fatos justificam a importância das redes sociais aos idosos; onde, devem ser iniciadas ações educativas que potenciem a participação cidadã do idoso. Nesse contexto, é dever do educador social orientar a população idosa sobre seus direitos e deveres, promovendo educação em saúde de forma inclusiva e integradora (COSTA FB e TERRA NL, 2012). Antes da intervenção, foram feitas perguntas pontuadas préestabelecidas como, "satisfação com o que alcançou na vida, satisfação com uso de tempo, satisfação com nível de atividade e satisfação com participações na comunidade". Partindo disso, o educador social convidado pelo grupo a palestrar aos idosos, trabalhou em cima dessas questões, por meio de palestras educativas sobre temas fundamentais à essa população, como "Sexualidade na terceira idade, repercussão do Alzheimer, Direitos e deveres dos idosos, Importância do apoio familiar à população geriátrica, Influência da atividade física na saúde do idoso, Repercussão da alimentação balanceada", etc., o resultado obtido foi um aumento significativo da participação dos idosos na comunidade, no ambiente familiar e de autoconhecimento.

Uma das dificuldades encontradas durante a realização do projeto foi a quantidade elevada de idosos que possuem restrições relacionadas à sua qualidade de vida que limitaram seu comparecimento à Unidade Básica de Saúde, como, doenças osteoarticulares, dificuldade de locomoção ou ausência de acompanhante para consultas ou grupos de apoio. Durante a pesquisa, foram incluídos apenas idosos que pudessem comparecer fisicamente à Unidade Básica de Saúde. Outra limitação se refere à não obtenção da amostra total esperada no início do projeto, visto que durante a realização das ações de intervenção iniciais, um número maior de idoso do que o usado para verificação dos resultados comparecia à unidade, entretanto, essa parcela não demonstrou assiduidade durante as reuniões, por esse fato, foram retiradas da amostra final. Algo que exprime a probabilidade de que algumas diferenças significantes possam não ter sido evidenciadas em função do baixo poder do estudo (WALTER MIMT, 2010).

Quanto à religião, dentre as características que definiram o perfil da amostra avaliada, observou-se que $100 \%$ dos idosos possuíam uma religião e eram ativos nela, o que corrobora com a ideia de que as práticas religiosas podem ser uma forma de potencializar os sentimentos de autonomia, satisfação e bem-estar. As principais religiões referidas foram: católica $(53,6 \%)$, evangélico $(39,3 \%)$, seguido de espirita $(7,1 \%)$, o que coincide com o estudo realizado em São Paulo no ano de 2011, o qual havia uma incidência significante de pacientes idosos católicos, mostrando que a religião estava relacionada diretamente com a adesão ao tratamento e recuperação de doenças (GUTZ L, CAMARGO BV, 2013).

Além do mais, a população idosa se remete a ampla probabilidade de eventos de perdas, mudanças físicas, psicológicas, momentos de ociosidade e maiores pensamentos sobre morte. Dessa forma, há a aproximação com a espiritualidade, principalmente idosos com 80 anos, que estabelecem ideias como, (1) conexão com Deus; (2) transcendência da existência mundana; (3) qualidade de pensamento: importância da honestidade no convívio interpessoal; e (4) responsabilidade humana: diante de escolhas e possíveis consequências nessa vida (SANTOS TBG, 2010).

O Mini Exame do Estado Mental (MEEM), por ser um teste amplamente utilizado em todo o mundo é considerado um instrumento com boa consistência interna para a avaliação da função cognitiva e rastreamento de evidências demenciais em idosos (NUNES CAN, 2017).

Segundo esse estudo, após a intervenção houve uma evolução no perfil cognitivo dos idosos, sendo os com defeito cognitivo na primeira avaliação $(35,7 \%)$ resultou em queda na segunda avaliação $(21,4 \%)$; já os 
que não apresentaram defeito cognitivo na primeira avaliação (64,3\%) indicou acréscimo na segunda avaliação (78,6\%) (Tabela 4). Semelhantemente, o estudo da Escola Superior de Saúde no Instituto Politécnico de Bragança, teve como resultados obtidos no MEEM, relativo ao perfil cognitivo, funcional e níveis de sintomatologia depressiva, uma média na avaliação inicial de 23,30 e, posteriormente, na avaliação final de 27,40, apresentando seguimentos benéficos Concluiu-se, também, que a variável escolaridade influencia no escore cognitivo, já que $89,6 \%$ dos idosos integrantes possuem um nível de pelo menos quatro anos de estudo formal. Dessa forma, havendo uma discrepância com o estudo de Juliana Costa, o qual os idosos estudados apresentaram um baixo nível de escolaridade, com uma proporção de $91 \%$ de idosos com menos de quatro anos de estudo (MACHADO JC et al, 2011).

\section{CONCLUSÃO}

Os resultados obtidos no estudo sugerem que a grande prevalência de doenças crônicas e a insatisfação com a qualidade de vida são alguns dos fatores que diminuem a independência e a autonomia dos idosos, sendo necessária uma rede de apoio efetiva para essa população. Em vista dos argumentos apresentados, conclui-se que o projeto de intervenção obteve resultados satisfatórios, alcançando uma verdadeira integração comunitária entre idosos da USF Canal da Visconde, maior integração familiar e manutenção capacidade funcional, por meio da participação nos grupos de apoio. Portanto, essas ações de fortalecimento socio familiar e político da população idosa são fundamentais para melhorar o desempenho cognitivo, os índices de depressão e até mesmo o declínio funcional.

\section{REFERÊNCIAS}

1. ALBERTE JS, et al. Qualidade de vida e variáveis associadas ao envelhecimento patológico. Rev Soc Bras Clin Med., 2015;13(1):32-9.

2. ALBUQUERQUE AG, et al. Capacidade funcional e linguagem de idosos não participantes e participantes de grupos de intervenção multidisciplinar na atenção primária à saúde. Rev. CEFAC, São Paulo, 2012; 14(5): 952-962.

3. AMARAL LF, et al. Perfil do apoio social de idosos no município de Natal, Estado do Rio Grande do Norte, Brasil, $2010-2011$. Epidemiol. Serv. Saúde, 2013; 22(2):335-346.

4. BORGES MCM. O idoso e as políticas públicas e sociais no Brasil. As múltiplas faces da velhice no Brasil, 2006; 79-104.

5. COSTA FB, TERRA NL. Espiritualidade, religiosidade e qualidade de vida em idosos. Revista Geriatria e Gerontologia, 2012; 173-178.

6. FERREIRA FPC, et al. Serviços de atenção ao idoso e estratégias de cuidado domiciliares e institucionais. Rev. bras. geriatr. gerontol., 2014; 17(4): 911-926.

7. GARCIA MAA, et al. Atenção à saúde em grupos sob a perspectiva dos idosos. Rev. Latino-Americana de Enfermagem, 2006; 14(2):175-82.

8. GUIMARÃES RA, AMÉLIO SAA. rede social e os tipos de apoio recebidos por idosos institucionalizados. Rev. bras. geriatr. Gerontol., 2018; 16(1): 159-170.

9. GUTZ L, CAMARGO BV. Espiritualidade entre idosos mais velhos: um estudo de representações sociais. Rev. bras. geriatr. gerontol., 2013; 16(4): 793-804.

10. LEITÃO MCM, et al. Redes de apoio social e de suporte social e envelhecimento ativo. International Journal of Developmental and Educational Psychology, 2016; 1(1): 293-303.

11. MACHADO JC, et al. Declínio cognitivo de idosos e sua associação com fatores epidemiológicos em Viçosa, Minas Gerais. Rev. bras. geriatr. gerontol., 2011; 14(1): 109-121.

12. MOLINI-AVEJONAS DR, et al. Fonoaudiologia e Núcleos de Apoio à Saúde da Família: conceitos e referências. Rev Soc Bras Fonoaudiol., 2010;15(3):465-74.

13. NUNES CAN. Impacto de um Programa de Treino Cognitivo em Idosos Institucionalizados. Diss ertação (Mestrado em Enfermagem de Reabilitação). Escola Superior de Saúde de Bragança, 2017; 100p.

14. OLIVEIRA LPBA, MENEZES MP. Representações de fragilidade para idosos no contexto da estratégia saúde da família. Texto Contexto Enferm, 2012; 20(2):301-9.

15. PAOLINI KS. Desafios da inclusão do idoso no mercado de trabalho. Revista Brasileira de Medicina do Trabalho, 2016; 14(2): 177-82.

16. RIBEIRO GG, et al. Perspectivas sobre a aposentadoria e o trabalho na pós aposentadoria na terceira idade: revisão da literatura brasileira entre 1994 e 2014. XIV SEPA - Seminário Estudantil de Produção Acadêmica, UNIFACS, 2015.

17. SANTOS CS, et al. Avaliação da confiabilidade do Mini Exame do Estado Mental em idosos e associação com variáveis sociodemográficas. Cogitare Enfermagem, 2010; 15(3): 406-12.

18. SILVA JVF, et al. relação entre o envelhecimento populacional e as doenças crônicas não transmissíveis: sério desafio de saúde pública. Cadernos de graduação: Ciências Biológicas e da Saúde, 2015;2(3): 91-100.

19. VILELA ABA, et al. Envelhecimento bem-sucedido: representação de idosos. Revista Saúde Com., 2006; 2(2): 101-14.

20. WALTER MIMT. A dualidade na inserção política, social e familiar do idoso: estudo comparado dos casos de Brasil, Espanha e Estados Unidos. Centro de pesquisa de opinião pública. 2010; 186-219. 